\title{
Near-field Raman Spectroscopy using the Local Field-Enhancement Technique
}

\author{
L. Novotny and A. Hartschuh \\ The Institute of Optics, University of Rochester, Rochester, NY 14627 \\ (585)275-5767, (585)244-4936, novotny@optics.rochester.edu
}

Spectroscopic methods with high spatial resolution are essential for understanding the physical and chemical properties of nanoscale materials including biological proteins, semiconductor quantum structures, and nanocomposite materials. Unfortunately, the spatial resolution of conventional optical techniques is limited by the diffraction barrier, typically more than an order of magnitude larger than characteristic length-scales of most nanoscale systems. Recently, near-field optical microscopy $[1,2]$ has extended the range of optical measurements beyond the diffraction limit and stimulated interests in many disciplines [3].

In the most widely adapted aperture approach [4], light is sent down an aluminum-coated fiber tip of which the foremost end is left uncoated to form a small aperture. Unfortunately, only a tiny fraction of the light coupled into the fiber is emitted through the aperture because of the cut-off of propagation of the waveguide modes. The low light throughput and the finite skin depth of the metal are the limiting factors for resolution. Nowadays it is doubted that an artifact-free resolution of $50 \mathrm{~nm}$ will be surpassed by the aperture technique. However, higher resolutions are required for various investigations in nanoscale physics.

To overcome the limitation of the aperture technique, we have recently introduced a new near-field optical technique [5] that makes use of the strongly enhanced electric field close to a sharply pointed metal tip under laser illumination [c.f. Fig. 1a]. Depending on the tip shape, material and polarization conditions, the energy density close to the metal tip can be strongly enhanced over the energy density of the illuminating laser light $[6,7]$. The tip is held a few nanometers above the sample surface so that a highly localized interaction between the enhanced field and the sample is achieved. The method has been successfully applied for the imaging of photosynthetic membranes and for the localization of photoactive proteins [5] to probe highly confined fields as encountered in strongly focused laser beams [8], and recently to nanoscale vibrational
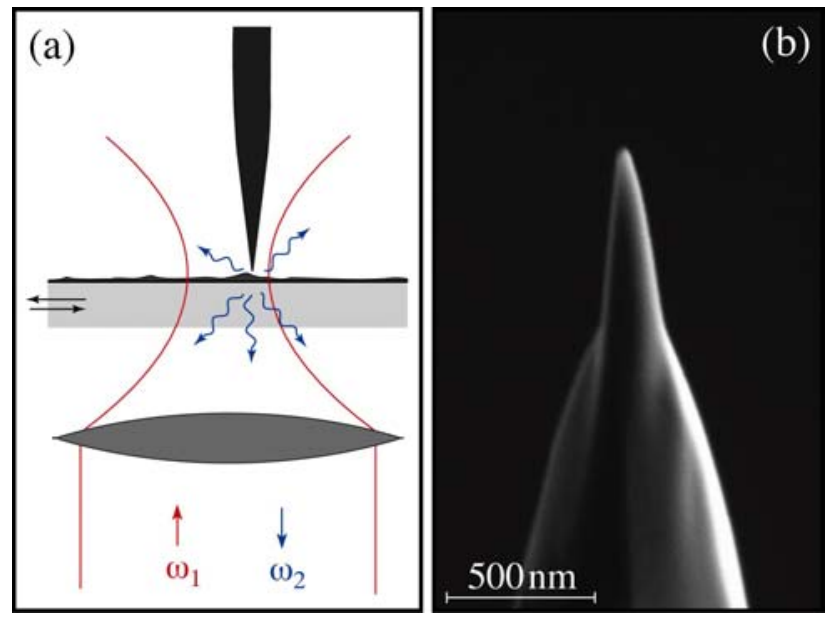

Fig. 1. Light confinement based on the field enhancement effect. (a) A laser beam is focused on a sample surface and a sharply pointed metal tip is positioned into the laser focus. The enhanced fields at the tip locally interact with the sample surface thereby exciting a spectroscopic response that is collected by the same objective and directed on a detector. (b) SEM image of a typical metal tip fabricated by electrochemical etching and subsequent focused ion beam milling. 

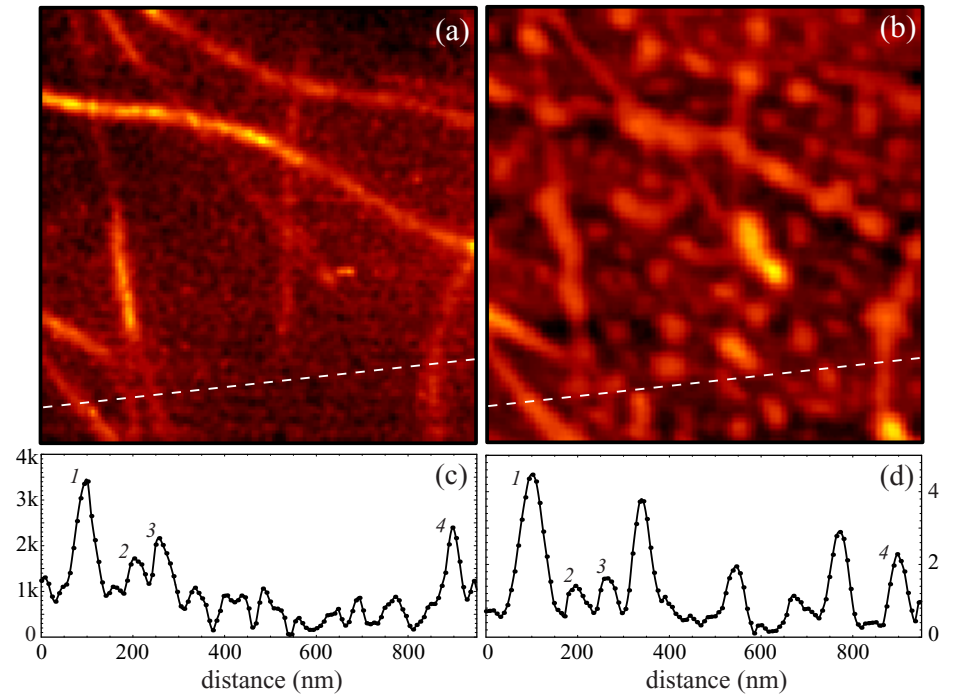

Fig. 2. Simultaneous near-field Raman image (a) and topographic image (b) of SWNTs grown by $C V D$ on glass. Scan area $1 \times 1 \mu \mathrm{m}$. The Raman image is acquired by detecting the intensity of the $G$ 'band of $S W N T s$ upon laser excitation at $633 \mathrm{~nm}$. No Raman scattering signal is detected from humidity related circular features present in the topographic image. (c) and (d) are cross sections taken along the indicated dashed lines. Labels $1-4$ mark the positions of the SWNTs and the vertical units are photon counts per second and nanometers, respectively. From [9].

spectroscopy of carbon nanotubes [9] [c.f. Fig. 2].

The latter approach makes use of the well known effect of surface enhanced Raman scattering (SERS). The results shown in Fig. 2 clearly demonstrate that it is possible to locally enhance the Raman scattering crosssection of a sample placed near the tip. The vibrational modes (G'-band, $\nu=2615 \mathrm{~cm}^{-1}$ ) of individual singlewalled carbon nanotubes (SWNT) were imaged with a resolution better than $20 \mathrm{~nm}$. Since all molecules have a unique fingerprint in form of a vibrational spectrum, the near-field Raman technique has great potential for becoming a routine tool for the chemical analysis of surfaces and subsurface structures.

In this presentation, we will present an introduction to the local field enhancement technique and discuss recent experimental results.

\section{References}

1. D.W. Pohl, W.Denk, and M.Lanz, "Optical stethoscopy: Image recording with resolution lambda/20," Appl. Phys. Lett., vol.44, pp.651-653, 1984 .

2. A.Lewis, M.Isaacson, A.Harootunian, and A.Muray, "Development of a 500 A resolution light microscope," Ultramicroscopy, vol.13, pp.227-231, 1984 .

3. For a review, see, R. Dunn, "Near-field scanning optical microscopy," Chemical Reviews, vol.99, pp.2891-2928, 1999.

4. E.Betzig and J.K. Trautman, "Near-field optics: Microscopy, spectroscopy, and surface modification beyond the diffraction limit," Science, vol.257, pp.189-195, 1992.

5. E. J. Sanchez, L. Novotny, and X. S. Xie, "Near-field fluorescence microscopy based on two-photon excitation with metal tips," Phys. Rev. Lett., vol.82, pp.4014-4017, 1999.

6. J. Wessel, "Surface-enhanced optical microscopy," J. Opt. Soc. Am. B, vol. 2, pp. 1538-1540, 1985.

7. L.Novotny, E.J. Sanchez, and X.S. Xie, "Near-field optical imaging using metal tips illuminated by higher-order HermiteGaussian beams," Ultramicroscopy, vol.71, pp.21-29, 1998.

8. A. Bouhelier, M. Beversluis, A. Hartschuh, and L. Novotny, "Near-field second-harmonic generation induced by local field enhancement," Phys. Rev. Lett., in press, 2002.

9. A. Hartschuh, E. J. Sanchez, X. S. Xie, and L. Novotny, "High-resolution near-field Raman microscopy of single-walled carbon nanotubes," Phys. Rev. Lett, submitted, 2002. 